\title{
Bereavement management during COVID-19 Pandemic: One size may not fit all!
}

Ashwin Rammohan ${ }^{1}$, Priya Ramachandran ${ }^{1,2}$, Mohamed Rela ${ }^{1}$

${ }^{1}$ The Institute of Liver Disease \& Transplantation, Dr. Rela Institute \& Medical Centre, Bharath Institute of Higher Education \& Research, Chennai, India

${ }^{2}$ The Ray of Light Foundation, Kanchi Kamakoti CHILDS Trust Hospital, CHILDS Trust Medical Research Foundation, Chennai, India

T he way in which individuals react to the death of a loved one varies across cultures. The coping mechanisms to this grief however, remain remarkably constant. Irrespective of race, religion or culture death is almost universally followed by a funeral service or ritual [1]. Across the globe, communities have developed these rites to enable individuals and families cope and deal with the loss of their loved one. A funeral ritual allows for a culturally accepted expression of emotions, emphasizing the irreversibility of death. It also initiates the recovery processes of continuity, transition and transformation [2].

While statistics on the number of deceased are readily available, the grief of losing a loved one remains intangible. During this COVID-19 pandemic, strict regulations are in place for the management of the deceased and performance of last rites. Consequently, family members may not have the opportunity to achieve closure by resolving "unfinished business". This is likely to result in a prolonged grief disorder.
Deaths during communicable disease epidemics are even more distressing as they defy the concepts of an "ideal death". Family members may not have the opportunity to achieve closure by resolving "unfinished business". The pain and guilt of not physically seeing or being with their loved ones during their suffering exacerbates the grief. Furthermore, a lack of social recognition with impaired support system along with the absence of last rites results in a state of "disenfranchised grief". This is likely to result in a prolonged grief disorder, a condition which imperils the physical and psychological well-being of an individual [1,3].

The Ebola and Marburg virus disease epidemics were examples where strict regulations were in place for the management of the deceased and performance of last rites [4]. The rationale behind such strict guidelines were among others factors, a high case fatality rate observed in those who had prolonged contact with the corpse. Funerals also posed a substantial risk for transmission due to other reasons. First, an increased viral load was noted in the non-survivors more so during the terminal stages of the disease and this intuitively translated to a higher risk of transmission. Second, traditional practices across cultures included close contact with the body including washing and other preparations for public display. This meant that there was a prolonged period of contact of individuals with the corpse. Lastly, it is customary for these last rites to be well attended by family, friends and people within the community. Attendance was usually important to demonstrate deference and to establish socio-political and financial rights [1]. These attendees could then establish new chains of transmission, leading to an unchecked transmission of the virus.

While statistics on the number of deceased are readily available during an epidemic, the grief of a loved one remains intangible. Moreover, with the COVID-19 pandemic continuing unabated and due to the purported contagious nature of the virus, hospital rules prohibit family's access to the deceased [3,5]. For those dying in 
These regulations while pragmatic, may not be the most durable long-term models of helping people cope with bereavement. We present a compelling argument to revisit these dogmatic guidelines to allow the bereaved return to a more conventional process of coping with grief.

overwhelmed facilities, bodies may not be treated with the dignity they would ordinarily receive. With beleaguered staff quickly making beds available for new patients, dead bodies may end up being piled in corridors or trucks and disposed of without the family getting to see them. The WHO and Governments have placed restrictions on performance of last rites based on previous experience with highly communicable fatal diseases [6,7]. Main highlights of the guidelines include rules for removal of the body from the isolation area, handling of dead body in the mortuary and at the crematorium/burial ground. There is also a wide variation in the actual enforcement of these regulations. In the majority of cases, there is a tendency towards a much stricter enforcement of isolation procedures denying the relatives any form of closure. Some countries have laid down stringent guidelines which extend beyond the simple concept of controlling the spread of infection. For example, the Srilankan Government dictates that all COVID-19 deaths be compulsorily cremated [8]. Online memorial services, virtual support groups, telephonic grief counselling and other innovations may provide short-term bereavement support for survivors of COVID-19 deaths [3,5]. While pragmatic, these may not be the most durable long-term models of helping people cope with bereavement. Moreover, the opportunity to respect cultures, traditions and the need to provide comfort to those left behind remain incomplete.

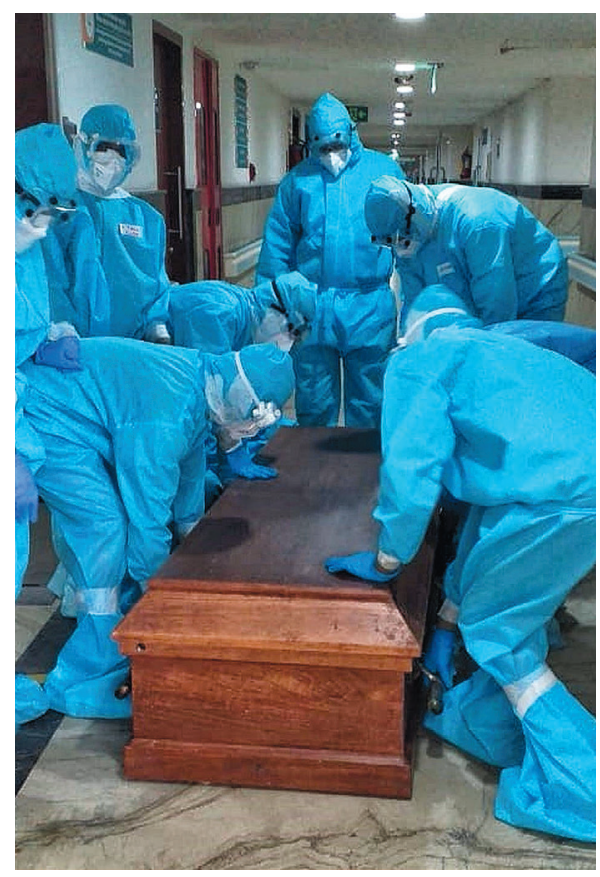

Photo: Hospital authorities in protective suits follow strict precautions in the management of the mortal remains of a COVID-19 patient (from the author's collection, used with permission).
With the pandemic raging on especially in the developing world, it is important to reassess the infectivity quotient of COVID-19 among the deceased. There are no models or real-life situations to objectively define this metric. Post-mortem studies have shown only autopsied lung tissue and any aerosolised bodily fluid to contain the infective particles [6]. Currently, regulations based on previous experiences with other highly communicable diseases like Ebola and Marburg virus have been used to define the radical and stringent means of deceased disposal. In this context, there exists important differences between COVID-19 deaths and other communicable diseases which need highlighting. Most of the COVID-19 deaths are due a dysregulated immune system rather than a direct cytopathic effect of the virus [9]. Most of those dying also spend a considerable time (2 to 3 weeks) in hospital before succumbing and it may even be longer from the onset of symptoms [9]. This would mean that they might not actually harbour viable virus at the time of death. While patients who recover from mild to moderate disease are allowed to return to work after 2 weeks of onset of illness, the regulations for management of deceased are still stringent, leaving a question which begs answering: Do the current deceased management regulations which are based on other more fatal and virulent communicable disease need to be revisited with a fresh perspective to allow for a return from this virtually charged "new normal" to the conventional performance of rites [10]?

Human resilience in the face of adversity will ultimately help the bereaved cope with grief. However, an objective method to evaluate the need for adopting innovative modes of support need to be assessed before embracing them as a part of the forbearance.

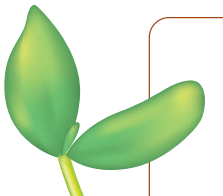

Acknowledgments: We thank all the Gansu public health staff who did the epidemiological investigation of the COVID-19 and the medical staff for their efforts in the treatment of patients.

Disclaimer: The views expressed in the submitted article are our own and not an official position of the institution. And the research has been approved by the academic committee.

Funding: This article was supported by Social Science Planning Major Project of Gansu Province (Grant No. 20ZD017).

Authorship contributions: All authors contributed to the conceptualization, writing, and revisions of the paper.

Competing interests: The authors completed the ICMJE Unified Competing Interest form (available upon request from the corresponding author), and declare no conflicts of interest.
} 
1 Lobar SL, Youngblut JM, Brooten D. Cross-cultural beliefs, ceremonies, and rituals surrounding death of a loved one. Pediatr Nurs. 2006;32:44-50. Medline:16572538

2 Li J, Tendeiro JN, Stroebe M. Guilt in bereavement: Its relationship with complicated grief and depression. Int J Psychol. 2019;54:454-61. Medline:29508381 doi:10.1002/ijop.12483

3 Wang SSY, Teo WZY, Yee CW, Chai YW. Pursuing a Good Death in the Time of COVID-19. J Palliat Med. 2020;23:754-5. Medline:32311289 doi:10.1089/jpm.2020.0198

4 World Health Organization. Field situation: How to conduct safe and dignified burial of a patient who has died from suspected or confirmed Ebola virus disease. Geneva: WHO; 2014.

5 Carr D, Boerner K, Moorman S. Bereavement in the Time of Coronavirus: Unprecedented Challenges Demand Novel Interventions. J Aging Soc Policy. 2020;32:425-31. Medline:32419667 doi:10.1080/08959420.2020.1764320

6 World Health Organization. Infection prevention and control for the safe management of a dead body in the context of COVID-19: interim guidance. 24 March 2020. Geneva: WHO; 2020.

7 World Health Organization. Practical considerations and recommendations for religious leaders and faith-based communities in the context of COVID-19: interim guidance. 7 April 2020. Geneva: WHO; 2020.

8 Verma A. Explainer: COVID-19 Dead Body Management - India \& World. 2020. Available: https://factly.in/explainer-covid19-dead-body-management-india-world/. Accessed: 10 September 2020.

9 Lu L, Zhong W, Bian Z, et al. A comparison of mortality-related risk factors of COVID-19, SARS, and MERS: A systematic review and meta-analysis. J Infect. 2020.. Medline:32634459 doi:10.1016/j.jinf.2020.07.002

10 NHS. Coronavirus COVID-19 Workforce managers' guidance. 2020;39:1-18.

\section{Correspondence to:}

Dr. Ashwin Rammohan

The Institute of Liver Disease \&

Transplantation

Dr. Rela Institute \& Medical Centre

Bharath Institute of Higher Education

$\&$ Research

Chennai

India

ashwinrammohan@gmail.com 